\title{
Bottom-Up Nanofabrication with Extreme-Ultraviolet Light: Metal- Organic Frameworks on Patterned Monolayers
}

\author{
O. Lugier,* N. Thakur, L. Wu, M. Vockenhuber, Y. Ekinci, and S. Castellanos*
}

Cite This: ACS Appl. Mater. Interfaces 2021, 13, 43777-43786

Read Online

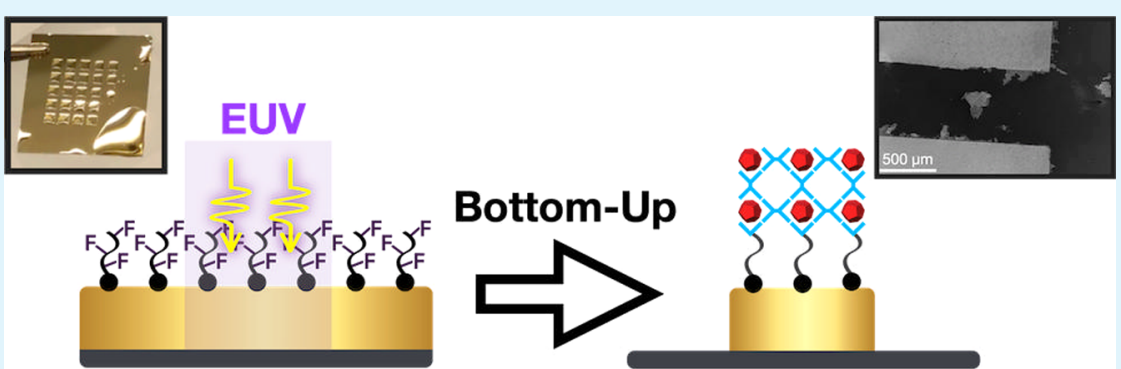

ABSTRACT: The fabrication of integrated circuits with ever smaller (sub-10 nm) features poses fundamental challenges in chemistry and materials science. As smaller nanostructures are fabricated, thinner layers of materials are required, and surfaces and interfaces gain a more important role in the formation of nanopatterns. We present a new bottom-up approach in which we use the high optical resolution offered by extreme ultraviolet (EUV) lithography to print patterns on self-assembled monolayers (SAMs). Upon radiation, low-energy electrons induce chemical changes in the SAM so that the projected image is transferred to the substrate surface. We use the chemical differences between exposed and unexposed regions to promote a selective growth of hybrid structures that can act as an etch-resistant layer for further pattern transfer or can be used as functional nanostructures. The EUV doses required to promote selective growth on exposed areas are close to industrial requirements. Furthermore, this method allows for the independent tuning of different steps in the EUV lithography process (photo-induced chemistry, spatially resolved chemical contrast, and formation of nanopatterns), an advantage over current resists, in which the same material plays all roles.

KEYWORDS: EUV lithography, self-assembled monolayers, HKUST, SURMOF growth, nanopatterns

\section{INTRODUCTION}

The semiconductor industry has become a point of intersection between economics and fundamental science as a result of a prediction known as Moore's law. ${ }^{1}$ The claim that the transistors' number in a computer chip had to double every 2 years now translates into a demand for integrated circuits of an $8 \mathrm{~nm}$ size by $2028 .^{2}$ To satisfy this economic interest, a long path of technological challenges culminated in the shift of the light source used in photolithography from a 193 to a $13.5 \mathrm{~nm}$ wavelength (extreme ultraviolet, EUV) in order to gain optical resolution. The release of the first devices branded "EUVinside" to the consumer market this year marked a significant milestone for EUV lithography (EUVL). However, manufacturing nanostructures of sub-10 nm dimensions with such energetic photons opens fundamental questions at the interface of chemistry, physics, and materials science.

Detailed knowledge on how EUV radiation interacts with matter is crucial to design photosensitive materials that resolve the projected images into nanopatterns, that is, photoresists. $^{3-7}$ In particular, the role of the electron cascade generated after photoionization, the mechanisms leading to the solubility switch, the influence of optional post-exposure procedures, and the blur resulting from the aforementioned processes are still being investigated. ${ }^{8,9}$ Furthermore, as the nanopatterns' size decreases, thinner photoresists are required to avoid high aspect ratios and pattern collapse, ${ }^{10,11}$ which has two main consequences from a scientific perspective: (1) a more efficient EUV absorptivity is needed; (2) the roles of interfaces (photoresist-vacuum and photoresist-wafer) become more important for the formation of the nanostructures. Consequently, new concepts and designs of systems that can effectively react to radiation with nanoscale spatial resolution should be explored and accompanied by investigations of the fundamental aspects of their functioning to fulfil the requirements of upcoming nanofabrication technologies.

Here, we propose an alternative to the classical photoresist concept by using an organic self-assembled monolayer (SAM)

Received: July 20, 2021

Accepted: August 18, 2021

Published: August 31, 2021 

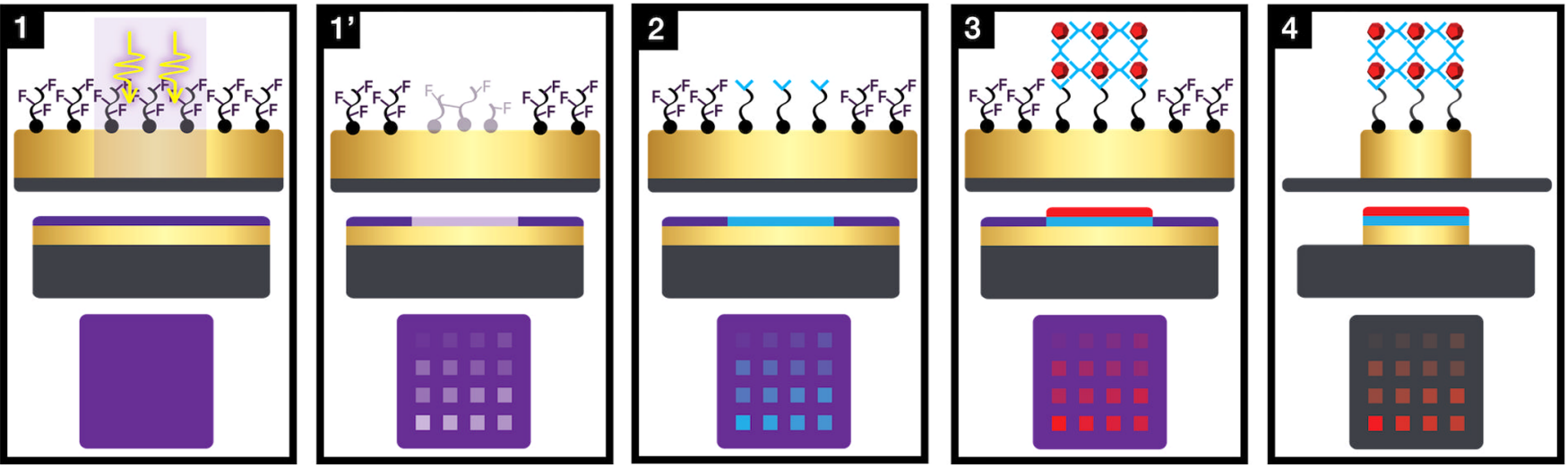

Figure 1. Schematic representation of a sample through each step of our bottom-up procedures for EUVL. (1) EUV exposure of the sample (1') structure of the sample post EUV irradiation; (2) selective exchange procedure by the replacement of the damaged areas of the SAM); (3) selective growth of SURMOF on the exchanged area; (4) etching of the areas not protected by the SURMOF. From top to bottom, the drawings represent the sample at the molecular level, its cross section, and its top view. In this work, steps 1-3 are investigated in detail, while step 4 was investigated as a proof of concept.
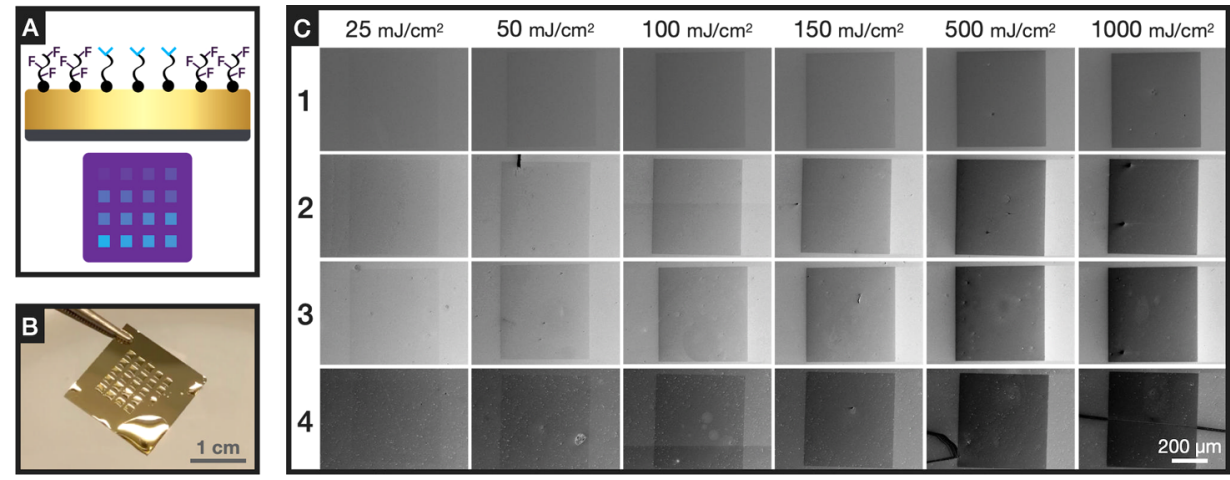

Figure 2. (A) Schematic representation of the samples (molecular level and top view) after the EUV-exposure (multidose) and exchange procedure of our bottom-up approach for EUVL. (B) Photograph of an HFDT-functionalized gold substrate after EUV exposure and exchange procedure in an MBA solution. The picture shows the sample a few seconds after being taken out of a solution of pure ethanol. The difference in hydrophobicity between pristine areas and EUV-exposed and MBA-exchanged areas $(1.7 \mathrm{~mm} \times 1.7 \mathrm{~mm}$ pads $)$ is revealed as the hydrophilic solvent drips away from the hydrophobic surfaces (unexchanged areas) but remains on hydrophilic surfaces (exchanged areas). (C) Compilation of SEM images of $500 \mu \mathrm{m} \times 500 \mu \mathrm{m}$ pads obtained on HFDT monolayers via EUV exposure and exchange in a solution of MBA or 4pyr. Different exchange procedures were conducted, such as (1) unexchanged, (2) pure ethanol for $48 \mathrm{~h}$, (3) 4pyr (6 mM) for $18 \mathrm{~h}$, and (4) MBA (6 mM) for $18 \mathrm{~h}$. In these images, the exposed/exchanged areas appear darker than the pristine background.

as a photoresist material in a novel bottom-up approach consisting of three main patterning steps: (1) exposure; (2) thiol exchange; and (3) selective growth (Figure 1). Monolayers of thiols are virtually transparent to $92 \mathrm{eV}$ photons $^{12}$ but sensitive to low energy electrons, which are generated after photoionization of the SAM and the substrate underneath. Upon irradiation (step 1), thiol SAMs undergo multiple chemical and structural changes, including packing disorder and decrease of the SAM stability, which create chemical differences between the exposed and unexposed areas at the surface of the functionalized substrate, referred to as chemical contrast. ${ }^{13-16}$ Thanks to this, the exposed areas can be selectively replaced by different thiol species through immersion of the substrate in a solution containing the new thiols (step 2, post-exposure exchange). This way, the chemical contrast of the patterns is enhanced, enabling subsequent surface modifications on exposed or unexposed areas. ${ }^{17}$ DNA, ${ }^{18,19}$ nanoparticles, ${ }^{20,21}$ quantum dots, ${ }^{22,23}$ and materials such as surface-mounted metal-organic frameworks (SURMOFs $)^{24}$ or perovskites ${ }^{25,26}$ can be deposited with various levels of selectivity on surfaces endowed with a spatially resolved chemical contrast. ${ }^{27}$ In this work, the chemical contrast obtained from the aforementioned procedure is used to promote the growth of a SURMOF on the exposed areas (step 3), which can later act as a protective layer for the etching step and enable the transfer of the pattern to the underlying substrate (step 4).

We observed that surface chemical contrast is obtained at doses near the industry target $\left(20-25 \mathrm{~mJ} / \mathrm{cm}^{2}\right)$. Furthermore, an advantage of this strategy is that each step can be tuned independently (the type of initial SAM and exchanging thiol and type of structure grown on the patterned monolayer), thus giving large room for optimization and opportunities for fundamental investigations. We believe the bottom-up approach presented here to be a potential breakthrough for building nanostructures over large areas in a cost-effective time.

Our approach can also be seen as a new way to fabricate nanostructures of functional SURMOFs for applications other than as an etch-protecting layer. MOFs in general are highly tunable materials provided with nanopores that can act as cavities for selective guest capture. In fact, they have shown promising properties for gas separation, catalysis, or in sensing 

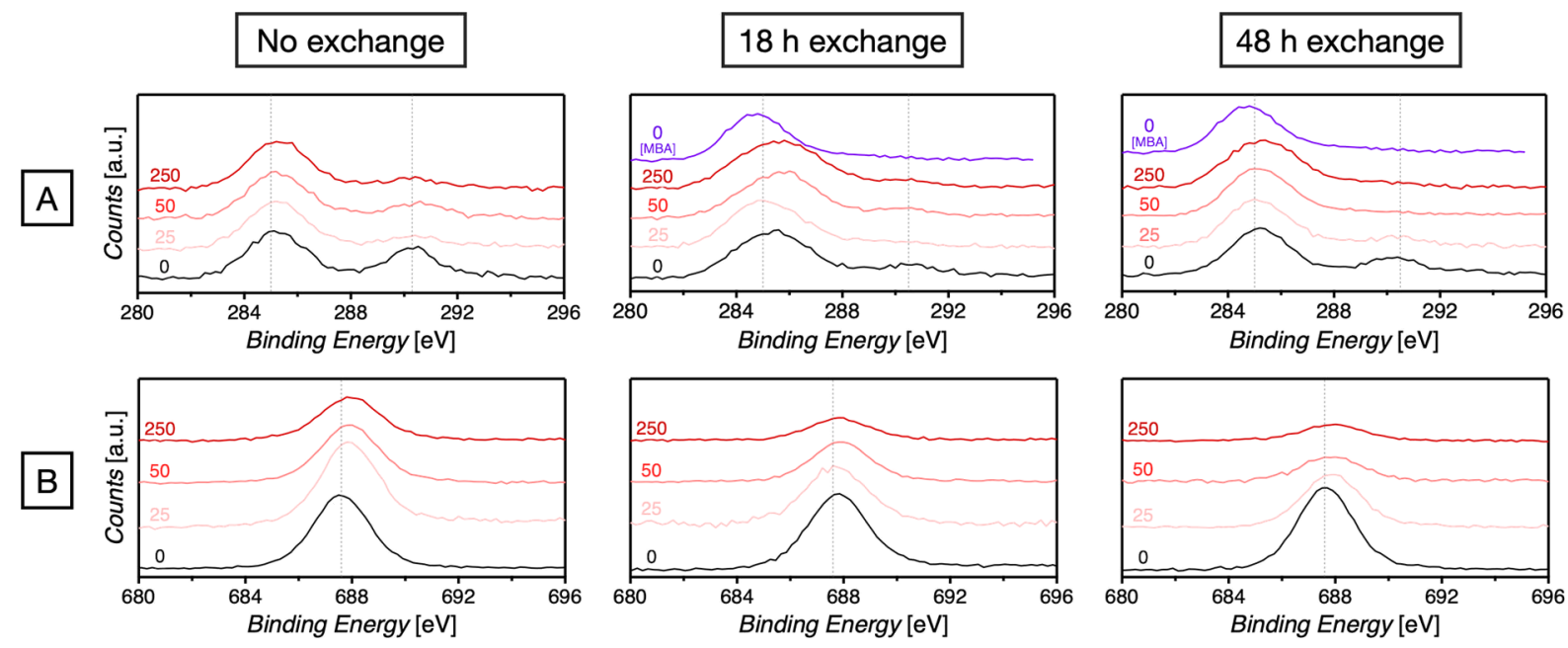

Figure 3. XPS spectra of the (A) C 1s region and the (B) F 1s region of the exposed samples (left), 18 h MBA-exchanged samples (middle), and 48 $\mathrm{h}$ MBA-exchanged samples (right) for various EUV doses. The numbers on each curve indicate the EUV dose received by the sample in $\mathrm{mJ} / \mathrm{cm}^{2}$. The C 1s spectrum of a pristine MBA-functionalized gold sample was added for comparison. The C 1s spectra (A) are normalized to their maximum intensity and the F 1s spectra (B) are normalized to the intensity of the gold signal of the substrate. Indicators are set at 285.0 and 290.3 $\mathrm{eV}$ for the $\mathrm{C} 1 \mathrm{~s}$ spectra (A) and at $687.5 \mathrm{eV}$ for the $\mathrm{F} 1 \mathrm{~s}$ spectra (B).

applications. ${ }^{28}$ Manufacturing nanostructures of these materials in high volume would allow their integration in devices, which has been a major challenge for the utilization of MOFs in the market. ${ }^{29}$ In the present work, we only work with a prototypical MOF as a proof of concept.

\section{RESULTS AND DISCUSSION}

Steps 1, 1' and 2: EUV Exposure and Post-Exposure Exchange. In our previous study, ${ }^{30}$ we showed that a thiol monolayer rich in fluorine, $(3,4,5,6,7,8,9,10)$-heptadecafluorodecanethiol (HFDT), on $\mathrm{Au}$ is chemically changed when exposed to EUV light and it was found that at a dose of 200 $\mathrm{mJ} / \mathrm{cm}^{2}, \sim 40 \%$ of fluorine is lost. The secondary electrons and holes generated upon EUV absorption trigger these chemical changes. Such changes in the chemical composition of the SAM necessarily introduce disorder in its packing, as reported in studies on the impact of low-energy electron irradiation on similar systems. ${ }^{13}$

Thiol monolayers are known to undergo desorption and exchange when immersed in a solution containing another thiol species, creating a mixed monolayer composed of both thiols. The rate of that desorption depends on various parameters such as the temperature, the type of solvent, the type of thiols used, and, most importantly, the quality of the packing of the initial monolayer. ${ }^{31}$ Regarding the influence of the packing, faster exchange rates are observed for increasing disorder, while defect-free regions remain integral. ${ }^{32}$

In this work, we study the exchange process of EUVirradiated HFDT monolayers using 4-mercaptomethyl benzoic acid (MBA) and 4-pyridylethyl mercaptan (4pyr) as replacing thiols. Their distinct chemical structures, as compared to HFDT, are expected to limit intermolecular interactions between the different SAMs and hence disfavor the formation of mixed domains and increase the chemical contrast between areas coated with HFDT and MBA or 4pyr. More importantly, both MBA and 4pyr act as nucleation sites for SURMOFs such as HKUST-1(Cu), an essential property for step 3 of our bottom-up approach (see below).

For a first assessment of the exchange procedure, a "hydrophobicity test" was performed following step 2 of the procedure (Figure 2a). SAMs composed of MBA and 4pyr have opposite wetting properties to the ones of HFDT. The former are hydrophilic (static water contact angle of $\sim 20$ and $\sim 60^{\circ}$, respectively), while perfluorinated SAMs are highly hydrophobic (static water contact angle of $\sim 120^{\circ}$ ). ${ }^{33}$ Soaking the surface of the samples with a polar solvent revealed the exchanged areas. The solvent drips away from the highly hydrophobic pristine monolayers, while small volumes remain confined on the hydrophilic domains (Figures 2b, S1 and videos in the Supporting Information). Although for certain processing conditions, this phenomenon was also observed in unexchanged samples, the dose threshold is significantly lowered with the exchange step (from $\sim 250$ to $\sim 75 \mathrm{~mJ} / \mathrm{cm}^{2}$ in samples with $500 \mu \mathrm{m} \times 500 \mu \mathrm{m}$ exposed areas). The hydrophobicity of HFDT monolayers originates from their high concentration of fluorine atoms. Thus, this hydrophobicity test supports our previous in-situ X-ray photoelectron spectroscopy (XPS) study, in which the F-loss was evidenced, ${ }^{30}$ and reveals on a macroscopic scale, the chemical contrast between unexposed and exposed and exchanged areas. This fact also evidences that the replacement of thiols on exposed areas by a different thiol species is favored.

Aiming at a more detailed inspection of the chemical contrast between exposed (and exchanged) and unexposed regions, scanning electron microscopy (SEM) was performed on selected samples (Figure 2c). We could image the different SAM domains present at the surface of the substrates. The exposed/exchanged areas appear darker than the unexposed background in the images. The observed trend is that a higher contrast is obtained for increasing the exposure doses, regardless of the exchange procedure. Even the reference samples, immersed in pure ethanol instead of a thiolcontaining solution, show contrasting exposed areas. This is another indication that EUV exposure induces chemical changes in the SAM even at relatively low doses $\left(25 \mathrm{~mJ} / \mathrm{cm}^{2}\right)$.

MBA-exchanged samples display a less marked contrast. We speculate that this phenomenon might originate from a faster exchange of the unexposed areas for this species, in agreement with the aforementioned reports on the stability of thiol SAMs in solutions. ${ }^{32}$ Another hint for this is the erosion of the $\mathrm{Au}$ 

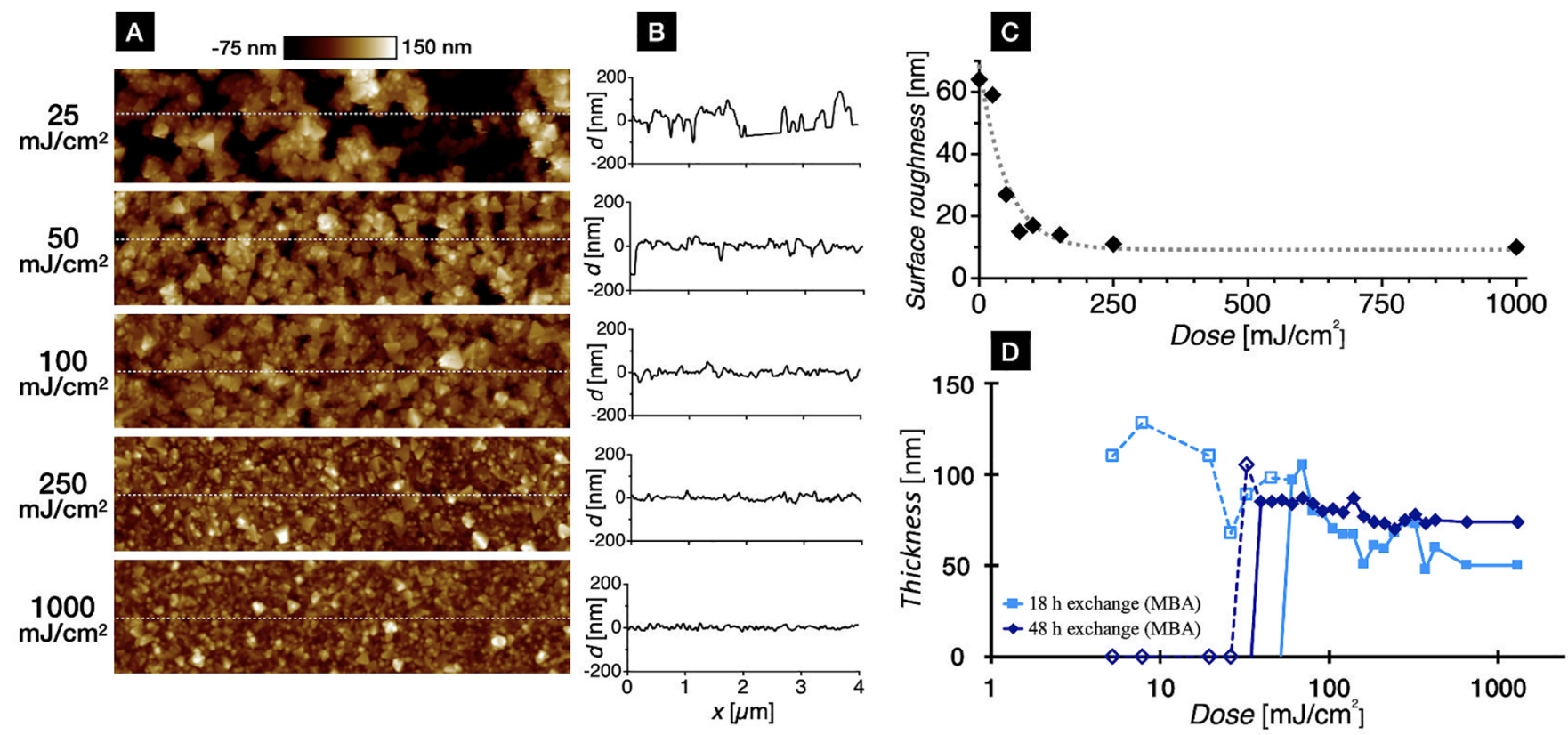

Figure 4. (A) AFM images $(4 \mu \mathrm{m} \times 1 \mu \mathrm{m}$; contact mode) of the thin films of HKUST-1(Cu) taken at the center of the pads (exchange procedure: 4PYR, $6 \mathrm{mM}, 48 \mathrm{~h}$ ). (B,C) Evolution of the surface roughness as a function of the initial EUV dose. The height profiles of (B) were taken along the white dotted lines shown in the AFM images. The values displayed in (C) were obtained from the entire AFM images. The exponential fit is shown as a gray dotted line on the graph. (D) Film's thickness as a function of the EUV dose (profilometer data). The hollow markers and dotted lines denote inhomogeneous HKUST-1 $(\mathrm{Cu})$ layers. The dose thresholds for the homogeneous layers are indicated by the continuous lines crossing the $\mathrm{x}$-axis at around 35 and $55 \mathrm{~mJ} / \mathrm{cm}^{2}$, respectively, for the $48 \mathrm{~h}$ procedure and $18 \mathrm{~h}$ procedure.

layer for these samples. We conclude that the degradation of the Au layer by this acidic thiol also is a consequence (and an accelerating factor) of the exchange on unexposed regions.

In general, darker regions originate from a lower density of backscattered electrons. Whether this is due to fewer electrons from the Au substrate escaping through the SAM (e.g., because the irradiated thiols generate a crosslinked C-network) or from fewer electrons being generated from the SAM (e.g., because of fewer F-atoms present) cannot be easily distinguished. Therefore, although SEM proved a good method to spot dose thresholds and qualitative trends, a quantitative analysis cannot be extracted from such data.

In an attempt to monitor the efficiency of the post-exposure exchange process, we used XPS on MBA-exchanged samples for various EUV doses and exchange procedures. In XPS, the position of the peaks in the binding energy scale reveals which elements are present in a sample, and small variations around that position indicate the oxidation state of each specific element (chemical shift). Monitoring the area of the peak in the $\mathrm{F}$ 1s region of the spectra allows for detecting changes in the concentration of fluorine. In addition, HFDT and MBA display distinctive features in the $\mathrm{C} 1 \mathrm{~s}$ spectrum as they contain $\mathrm{C}-\mathrm{F}$ and $\mathrm{COO}$ species, respectively, which give rise to peaks with different chemical shifts.

The C 1s spectra in Figure 3a are normalized in intensity to detect changes in the number of components with different chemical shifts in the $\mathrm{C} 1 \mathrm{~s}$ envelope as an indication of changes in the chemical composition of the samples after each step. Notice that the signal at $285 \mathrm{eV}$ is most likely mainly arising from adventitious carbon. The $F$ 1s spectra in Figure $3 b$ are normalized to the area of the $\mathrm{Au} 4 \mathrm{f}$ peak to capture the relative fluorine loss and show, as a function of the dose, how much of this loss is induced by the post-exposure exchange procedures.
The C 1s spectra after EUV irradiation show a decrease in the intensity of the CF2/CF3 components (ca. $290 \mathrm{eV}$ ). The F $1 \mathrm{~s}$ spectra show a significant decrease in intensity of the fluorine peak (687.6 eV, see also Figure S2 in the Supporting Information) in line with our previous in-situ irradiation studies, denoting the partial desorption of full HFDT molecules and/or outgassing of fluorine-containing fragments. ${ }^{30}$ The $+0.3 \mathrm{eV}$ blue shift of the $\mathrm{F} 1 \mathrm{~s}$ peak implies slight changes in the chemical environment of $\mathrm{F}$ atoms, presumably from structural reorganizations of the fluorocarbon chains of the monolayer upon exposure. ${ }^{34}$

The $18 \mathrm{~h}$ exchange procedure yields a widening of the $\mathrm{C} 1 \mathrm{~s}$ components assigned to $\mathrm{C}-\mathrm{H} \mathrm{sp} \mathrm{sp}^{2} / \mathrm{sp}^{3}$ carbon species as the dose increases. This is an indication that new carbon species are incorporated in the SAM, thus adding components on the higher energy range. We hypothesize that the exchanged samples consist of a mixture of partially fluorinated and unsaturated carbon chains and MBA thiols. Thus, although pure MBA does not display prominent features at this BE, such an assortment of molecules generates a distribution of intermolecular interactions that lead to a broadening and shift of the $\mathrm{C}$ 1s signal. In addition, the carboxylic carbons (COOs) of the MBA molecules have binding energies around $288 \mathrm{eV},{ }^{34,35}$ so that a certain contribution of these electrons is expected. We also observe a decrease in the intensity for the CF2/CF3 components and of the F 1s peak along with the dose increase. However, the $+0.3 \mathrm{eV}$ shift seems unaffected. This might indicate that some of the fluorine-containing products remain after exchange. The same trends are registered in the $48 \mathrm{~h}$ exchange procedure, yet with a more significant loss of fluorine content (Figure 3b).

The qualitative comparison of the chemical composition of the various systems provides valuable insights into the mechanisms that take place during the procedure. Combined 


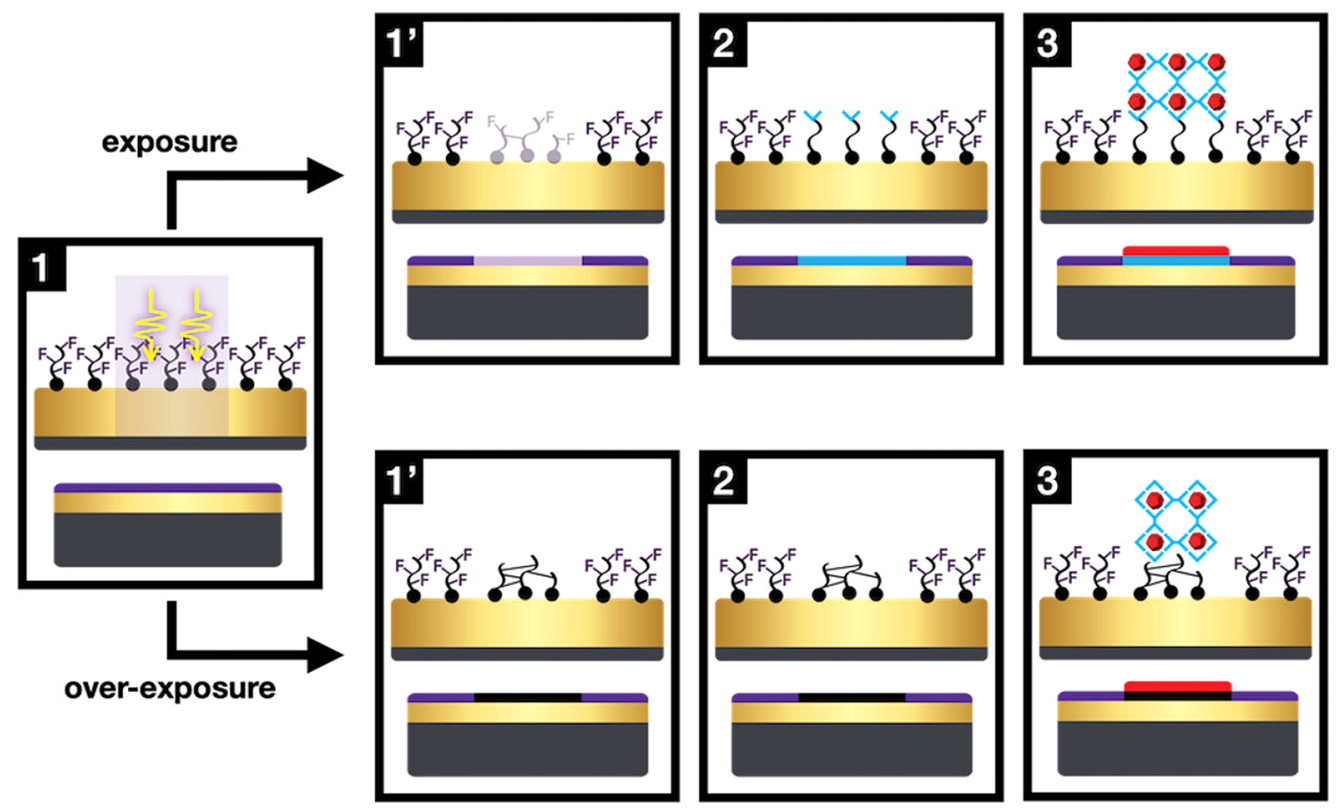

Figure 5. Schematic representations of a sample through the various steps of our bottom-up approach for EUVL. This figure illustrates the differences between samples exposed at an appropriate dose (top) and samples that were over-exposed (bottom).

with the previously discussed hydrophobicity test, SEM inspection, and our previous F-loss studies, ${ }^{30}$ these XPS results indicate that both desorption and incorporation of new species occur during the exchange procedure on areas irradiated by EUV photons.

Step 3: SURMOF Growth. For the final step of the bottom-up procedure, HKUST-1 $(\mathrm{Cu})$ is grown as a SURMOF on the surface of the EUV-exposed (and exchanged) samples via layer-by-layer (LbL) liquid phase epitaxy (LPE). ${ }^{36}$ Contrarily to pyridyl- and carboxyl-terminated SAMs, CF3terminated SAMs such as HFDT are known to be unsuitable for the growth of most SURMOFs, including SURMOFS of the HKUST-1 family. ${ }^{37}$ As a result, when an HFDTfunctionalized substrate that has been exposed to EUV (multiple pads with an increasing dose) is used for the LbLLPE step, HKUST grows preferably on the exposed areas. As discussed earlier, EUV irradiation of perfluorinated alkane thiols triggers the loss of fluorine and fluorine-containing fragments through bond cleavage and outgassing. In other words, exposing HFDT to increasing EUV doses depletes the SAM of its fluorine content, effectively transforming the perfluorinated thiols of such areas into alkane-/alkene-rich thiols and creating favorable domains for the growth of HKUST-1 $(\mathrm{Cu})$.

The effect is clearly enhanced when the exposed HFDT SAM is exchanged with 4 pyr or MBA (Figure S7 in the Supporting Information). That is, the SURMOF growth is favored at lower EUV doses if the new thiols are incorporated during an exchange procedure. The homogeneity of the SURMOF layers of the pads improves with increasing EUV dose. Usually, low doses yield colorless chips of transparent material randomly placed within the exposed area and high doses yield brown, fully homogeneous, and opaque thin films. The aspect of intermediate doses can vary depending on the size of the patterns, the exchange method, and the growth conditions. However, in general, it gives blue, mostly homogeneous and opaque layers of $\operatorname{HKUST-1}(\mathrm{Cu})$ (Figure S7 and S8 in the Supporting Information).
Profilometer and atomic force microscopy (AFM) reveal that the transparent films formed on areas exposed to medium doses are thicker and composed of bigger crystals than the opaque layers obtained when high EUV doses were used (Figure 4). As each MBA or 4pyr thiol is a potential nucleation point for the SURMOF, the number of nucleation points available for MOF crystals is correlated to the efficiency of the thiol exchange step. We hypothesize that the differences in thickness (Figure 4D) and roughness (Figure 4B,C) between layers grown on areas exposed to high and low doses stem from disparities in the availability of such nucleation sites at the surface. On high-dose areas where a higher number of photons hits the surface, the crystals nucleate closer to each other, resulting in an early coalescence and the formation of a smoother, more homogeneous film. However, with less nucleation points available over the same area, low dose patterns promote the development of discrete islands of material. Similar observations were reported on the properties of SURMOFs grown on seeded substrates, another synthetic method used to grow MOFs on a surface. ${ }^{38}$ In addition to the exposure dose, the thiol exchange time is also expected to have an impact on the number of nucleation points as the amount of the newly introduced thiol in the exposed areas and their packing density will depend on it. However, an exhaustive study on the effect of each of the growth parameters on the crystalline layer morphology is out of the scope of the current work.

It is worth mentioning that extensive crosslinking is expected to happen at high doses as the SAM experiences more fragmentation and desorption from sustained EUV irradiation. This might prevent the full exchange of the exposed thiol, as indicated by the presence of $\mathrm{F}$ after long exchange in the XPS spectra (Figure 3). The same phenomenon was reported on thiol SAMs following their exposure to high doses of lowenergy electrons. ${ }^{39}$ In this situation of over-exposure, the surface of the sample is expected to be covered by polymerized alkane or alkene species which form matrices bonded to the substrate at multiple points, thus disfavoring exchange with 

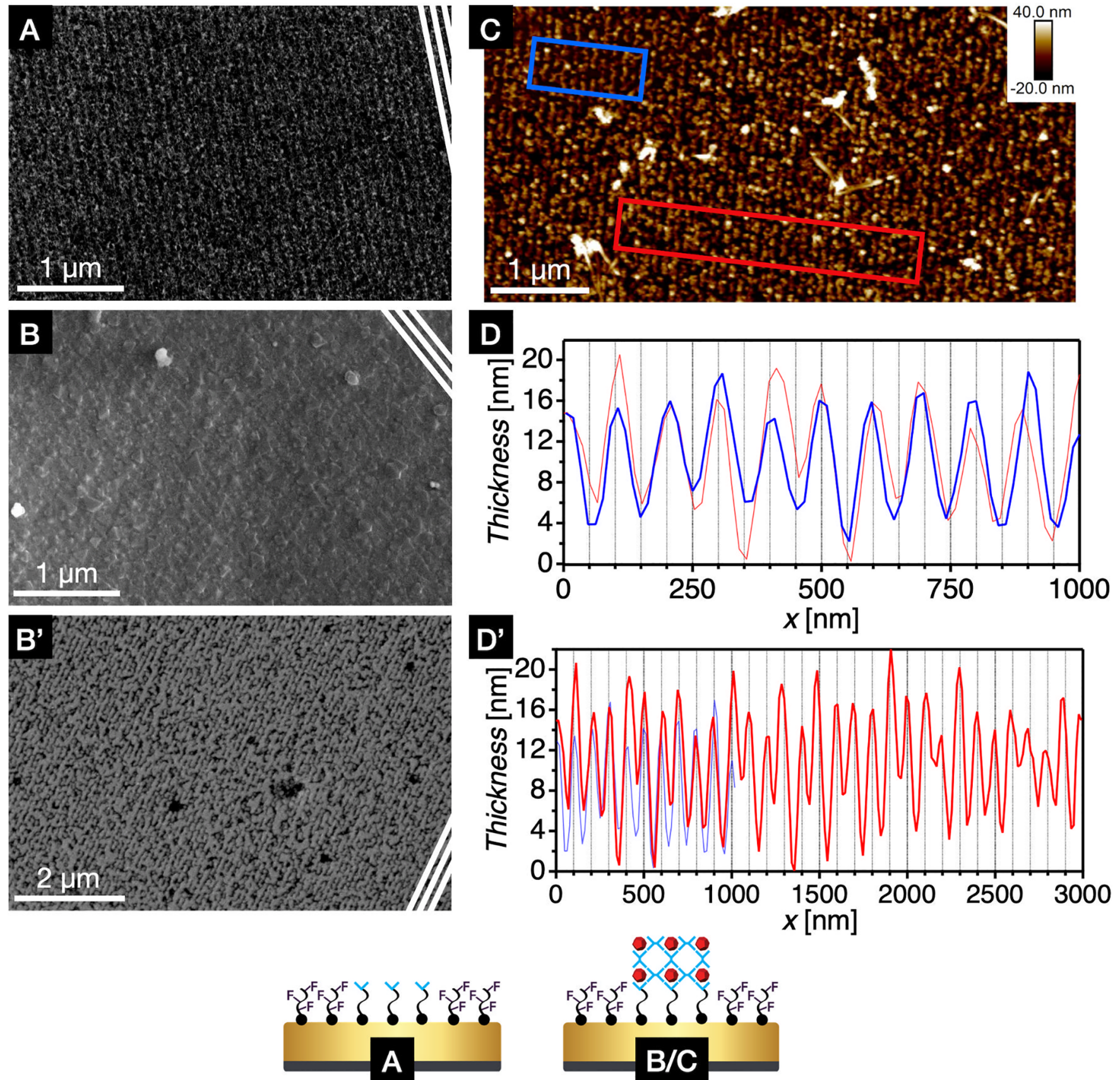

Figure 6. (A) Edited (contrast and brightness) SEM picture of line/space patterns (half-pitch: $50 \mathrm{~nm}$; dose: $500 \mathrm{~mJ} / \mathrm{cm} 2$ ) obtained via a EUV patterning and exchange procedure (MBA, $6 \mathrm{mM}, 48 \mathrm{~h}$ ) on a HFDT monolayer on Au. The original image is available in Figure S4 in the Supporting Information. (B, $\left.\mathrm{B}^{\prime}\right)$ SEM images of line/space patterns (half-pitch: $50 \mathrm{~nm}$; dose: $500 \mathrm{~mJ} / \mathrm{cm} 2$ ) in a thin film of HKUST-1(Cu) obtained with the following procedure: EUV patterning on a HFDT monolayer on Au, exchange in ethanol (MBA, $6 \mathrm{mM}, 48 \mathrm{~h})$, and $15 \mathrm{cycles}(\mathrm{B})$ or 7 cycles $\left(\mathrm{B}^{\prime}\right)$ of layer-by-layer LPE growth. (C) AFM images of line/space patterns from the same sample than $\left(\mathrm{B}^{\prime}\right)$. (D, $\left.\mathrm{D}^{\prime}\right)$ superposition of the height profile cross sections extracted from the areas of the same color code on (C). The grid lines have a pitch of $50 \mathrm{and} 100 \mathrm{~nm}$ in $\left(\mathrm{D}, \mathrm{D}^{\prime}\right)$, respectively. Both areas display identical patterns with the same pitch $(50 \mathrm{~nm})$ than the original EUV patterns. The dust particles seen on the surface are contamination from storage and transportation. The schemes at the bottom of the figure illustrate the state of the samples at the time of each measurement.

other thiols. Nonetheless, the chemical contrast created at such high doses between the now $\mathrm{CH}_{2}$-/ $\mathrm{CH}_{3}$-terminated exposed areas and the $\mathrm{CF}_{2}-/ \mathrm{CF}_{3}$-terminated pristine areas can be sufficient to enable preferential growth or deposition (Figure $5)$. For example, HKUST-1 $(\mathrm{Cu})$ has been shown to grow with remarkable efficiency on alkane-terminated SAM. ${ }^{40}$

Although preferential nucleation is obtained on exposed and exchanged areas, thin chips of material are also found on pristine domains. They are either weakly bonded to the substrate or sprouting from the edges of homogeneous SURMOF layers, sometimes bridging two adjacent exposed areas (Figure S3 in the Supporting Information). They are clearly not attached to the surface as sonication in a pure solvent removes them without apparent damage to HKUST$1(\mathrm{Cu})$ layers grown on exposed areas. Undesired deposition of 
MOF on unexposed areas could be avoided by further optimizing the SURMOF growth conditions.

Nanolithographic Performance. The thicknesses of the SURMOF layer grown on exposed samples after two different procedures ( $18 \mathrm{~h}$ and $48 \mathrm{~h}$ of the exchange step with MBA) as a function of dose are plotted in Figure 4D. This plot is the equivalent of the contrast curve of a photoresist in conventional lithography and provides information regarding the sensitivity of a resist material under specific conditions. Here, the key information is the minimum dose required to favor HKUST growth. We noticed that, under the tested conditions and for the specific exposed area sizes $\left(1.7 \times 1.7 \mathrm{~mm}^{2}\right)$, this dose threshold is ca. $7.5 \mathrm{~mJ} / \mathrm{cm}^{2}$ for the $18 \mathrm{~h}$ exchange (although the films remain mostly inhomogeneous up to ca. 50 $\mathrm{mJ} / \mathrm{cm}^{2}$ ) and ca. $40 \mathrm{~mJ} / \mathrm{cm}^{2}$ for the $48 \mathrm{~h}$ exchange one. The decrease in the dose threshold as the thiol-exchange time increases is attributed to a higher population of the thiol that favors growth in the exposed part and/or a better packing of the new thiol domain (see the XPS analysis above). This dose drop shows the versatility of this method since the tuning of the thiol-exchange time could decrease the EUV dose required to print nanoscaled features. In addition, other combinations of SAM pairs could also be used to further decrease the dose threshold toward values acceptable for cost-effective nanofabrication. For instance, we anticipate that using other thiols with hydrophobic tails (e.g., silanes) but with bulkier carbon backbones would decrease the packing efficiency of the initial patternable SAM. That would decrease the number of photoninduced reactions that are needed to switch the chemical contrast of the exposed area, thus leading to a lower dose. Following the same reasoning, smaller thiols that promote the MOF growth could diffuse more easily to the exposed areas. In addition, a tone inversion could be also considered, that is, starting with a SAM that enables the MOF growth on the unexposed area and replacing it with thiols that do not at the exposed areas.

Assuming a packing of 2-4 molecules of HFDT per square nanometer, 7.5 and $40 \mathrm{~mJ} / \mathrm{cm}^{2}$, respectively, correspond to 17 to 9 and 92 to 46 incident photons per molecule. This might appear as a rather inefficient process as a high number of photons per molecule is required for a usable chemical change. However, it should be noticed that the SAM has an EUV transmittance of ca. $98 \%$. Most photons are thus absorbed by the gold substrate and lead to an electron cascade in the $\mathrm{Au}$ layer, from which only the scaping electrons induce damage to the SAM on top. Taking into account that the inelastic mean free path of electrons of $80 \mathrm{eV}$ is estimated to be $\sim 5 \AA$ in $\mathrm{Au},{ }^{41,42}$ electrons generated in $\mathrm{Au}$ upon absorption of EUV photons would only escape from the topmost $1.5 \mathrm{~nm}$. This thin layer would absorb $7 \%$ of the photons that go through the SAM, meaning that overall, approximately only $9 \%$ of the incident photons are absorbed by the SAM/Au system. However, determining how many of these scaping electrons interact with the SAM is not straightforward. Therefore, the actual efficiency of the process is not yet known but can be further tuned using other substrates and monolayers. Remarkably, the dose thresholds for growth are close to the values targeted by the semiconductor industry $(20 \mathrm{~mJ} /$ $\left.\mathrm{cm}^{2}\right){ }^{43,44}$

Dense lines patterning was performed with EUV interference lithography as a preliminary investigation of the nanolithographic capability in terms of the resolution of the bottom-up approach in the studied conditions. EUV illumination of line/space patterns with various half-pitches (HPs) was projected on the HFDT monolayer, that is, 22, 30, 40, and $50 \mathrm{~nm}$ using different EUV doses (on the wafer), namely, 25, 50, 100, 250, and $500 \mathrm{~mJ} / \mathrm{cm}^{2}$. Only the HP $50 \mathrm{~nm}$ patterns on the HFDT monolayers exposed at $500 \mathrm{~mJ} / \mathrm{cm}^{2}$ are resolved enough to be observed with SEM after MBAexchange (Figure 6a). On 4pyr-exchanged samples, SEM images reveal the lines of HP $50 \mathrm{~nm}$ from $100 \mathrm{~mJ} / \mathrm{cm}^{2}$ onward, HP $40 \mathrm{~nm}$ from $250 \mathrm{~mJ} / \mathrm{cm}^{2}$ onward, and HP $22 \mathrm{~nm}$ for 500 $\mathrm{mJ} / \mathrm{cm}^{2}$ only (Figures S5 and S6 in the Supporting Information).

Unfortunately, the limited resolution of the SEM images, due to the invasive nature of SEM and the low contrast between exposed (and exchanged) lines and unexposed spaces (comprising HFDT), hinders the evaluation of the pattern quality. Therefore, defects related to photoelectron blur and defects such as bridging or line discontinuity cannot be properly assessed from these images, especially for the lowest pitches and doses. Nevertheless, these images confirm that nanometer-scale patterned SAMs can be obtained with this method.

After the exposure and exchange steps, HKUST-1(Cu) was grown on the line/space patterned monolayers to study whether the chemical contrast provided by the surface can be propagated to the SURMOF structure and study the impact of the growth step on the pattern resolution. On MBA-exchanged samples, for 15 cycles of LPE growth, SEM images show the crystals at the surface of the thin films arranged following a repetitive pattern of parallel lines (Figure 6B). The spatial alignment of individual crystals indicates that the SURMOF growth was influenced by the underlying patterned SAM. Distinct line/space patterns were obtained for the 7-cycle procedure (Figure $6 \mathrm{~B}^{\prime}, \mathrm{C}$ ) although the patterns suffered from low homogeneity and displayed line discontinuities. Nevertheless, the cross-sectional data obtained with AFM measurements (Figure 6D, $\mathrm{D}^{\prime}$ ) clearly show that the pattern transferred to the SURMOF perfectly matches the $50 \mathrm{~nm}$ half pitch of the original EUV pattern. An important contribution to the resolution loss observed for the 15 -cycle procedure is the crystal structure of HKUST-1 $(\mathrm{Cu})$. When anchoring points are present in the substrate, this MOF tends to grow in the [111] crystalline direction. This growth leads to the formation of cube-like crystals with one corner pointing upward, as it can be observed in the AFM images (Figure 4). This causes the horizontal expansion of the lines and their subsequent merging (Figure 6B), a phenomenon also observed between some patterns obtained via open frame exposures (Figure S3 in the Supporting Information). In addition, the size $(40-250 \mathrm{~nm})$ of the crystallites of HKUST-1 obtained from these synthetic conditions is larger than the printed HP. Using a different SURMOF platform with a preferred anisotropic growth, that is, pillar-layered MOFs such as DMOF $(\mathrm{Cu}, \mathrm{Co}, \mathrm{Zn}),{ }^{45}$ is also expected to improve the performance of the procedure.

Note that to use the bottom-up approach as an alternative to traditional photoresists, the primary objective of the material deposition for contrast enhancement is to provide an etch resistance for future pattern transfer. Thus, the most relevant threshold here is the minimal dose needed to obtain a homogeneous layer of HKUST deposited on the exposed areas, which stands at around $40 \mathrm{~mJ} / \mathrm{cm}^{2}$ for the conditions investigated in this work.

Step 4. Finally, as a proof of concept for step 4 of the procedure (Figure 1), we tested HKUST-1 $(\mathrm{Cu})$ as an etch- 
mask for the transfer of the pattern to the substrate, the ultimate role of a photoresist. Samples prepared with the bottom-up approach were etched in a potassium iodide solution, a common etching agent for Au. After the procedure, SEM images show that the gold layer remains only on the areas that were previously covered by MOF layers, while the pristine domains of the substrate are stripped. (Figure S9 in the Supporting Information).

\section{CONCLUSIONS}

In this work, we have demonstrated that surface chemical contrast can be attained by exposing monolayers of fluorinated thiols on Au substrates to EUV light at doses close to the industrial requirements for the fabrication of future integrated circuits using EUVL. Although the metrology of dense-line patterns on monolayers needs to be optimized, we observed the printing of $50 \mathrm{~nm}$ lines on such systems. Exchanging the damaged fluorinated thiols after EUV exposure with $\mathrm{N}$ terminated or COO-terminated thiols favors the selective growth of anchored MOFs on the exposed areas. Under the particular conditions used in the present work, the dose threshold for this process could be as low as $7.5 \mathrm{~mJ} / \mathrm{cm}^{2}$. However, we presume that at high doses, the crosslinking induced by EUV radiation on the fluorinated thiol is sufficient to promote such growth. The chosen SURMOF proved to act as an etch-protective layer for Au etching. We would also like to remark that the bottom-up method here presented can also be envisioned as a novel nanopatterning method for SURMOFs and, therefore, as a viable way of integrating SURMOFs in devices in high-volume manufacturing.

This bottom-up method will improve its efficiency and resolution capabilities, thanks to the high number of variables for optimization that it conveys. One could, for example, tune the experimental conditions of the exchange step to improve the pattern quality by increasing the purity of the SAM domains or select SURMOF species that grow more vertically, inducing less resolution loss. Even the core principles of the method can be tuned by choosing different types of monolayers, materials, or substrates. Finally, because our bottom-up approach stands out as compared to more conventional EUVL methods, we hope that its optimization and fine-tuning will unravel valuable insights into various processes of nanolithography.

\section{METHODS}

The chemical reagents used in this work were ordered from SigmaAldrich and used as such.

Sample Preparation and Thiol-Functionalization. Polycrystalline gold surfaces were prepared by sputter-coating (Leica EM ACE600) clean silicon substrates with a 5-nm adhesion layer of chromium followed by a 50 -nm layer of gold.

Initial functionalization of the gold surfaces was conducted by immersion in an ethanoic solution of HFDT at a concentration of 2 $\mathrm{mM}$ for $24 \mathrm{~h}$ following standard reported procedures. ${ }^{46}$ The hydrophobicity of the monolayers was determined using simple static water contact angle measurements. Typical values ranged from 100 to $110^{\circ}$.

The exchange of the EUV-exposed thiols was similarly conducted by immersion in ethanoic solutions of MBA or 4-pyridylethyl mercaptan at a concentration of $6 \mathrm{mM}$ for $18 \mathrm{~h}$ or $48 \mathrm{~h}$.

EUV Exposure. Open frame exposures (pads) were performed by exposing areas of $1.7 \times 1.7 \mathrm{~mm}^{2}$ (pinhole $70 \mu \mathrm{m}$ ) or $0.5 \times 0.5 \mathrm{~mm}^{2}$ (pinhole $30 \mu \mathrm{m}$ ), while line/space patterns were obtained via EUV interference lithography. Both pads and line/space patterns were exposed to a wide range of EUV doses. All EUV exposures were done at the XIL-II beamline at the Swiss Light Source (SLS) synchrotron in the Paul Scherrer Institute (PSI). ${ }^{47}$

Thiol Exchange. The exchange procedures consist in the immersion of the samples into a $6 \mathrm{mM}$ ethanolic solution of either 4-(mercaptomethyl)benzoic acid (MBA) or 4-pyridylethyl mercaptan (4pyr) at room temperature for 18 or $48 \mathrm{~h}$. After the procedure, the samples were gently rinsed with pure ethanol and dried under nitrogen flow. The reference samples were treated following the same procedure with pure ethanol solutions.

Imaging. The features on exposed-only or exposed-and-exchanged monolayers were characterized by SEM (FEI VERIOS 460) at energies of 2 or $5 \mathrm{keV}$ and beam currents of $0.1 \mathrm{nC}$ or $0.4 \mathrm{nC}$. The SEM images of grown SURMOFs were then taken at $5 \mathrm{keV}$ and a beam current of $100 \mathrm{nC}$.

AFM images were taken on a Bruker Scan-Assist atomic force microscope in contact mode with the silicon nitride Bruker Scan Assist-Air tips and the data treatment was carried out using Nanoscope software version 9.0. Typical scan rates were $1 \mathrm{~Hz}$. Scans were conducted with triangular Bruker Scan Assist Air tips of a nominal radius of $2 \mathrm{~nm}$, a nominal length of $115 \mu \mathrm{m}$, a nominal width of $25 \mu \mathrm{m}$, and a nominal spring constant of $0.4 \mathrm{~N} / \mathrm{m}$. Typical scan rates were $1 \mathrm{~Hz}$ and the resolution was $512 \times 512$ or $250 \times 250$.

SURMOF Growth. SURMOFs were grown using layer-by-layer LPE using a homemade automatized system with control over the temperature, time of immersion, stirring, and sonication. Up to six samples can be subjected to the synthetic procedure at the same time, allowing for simultaneous growth and more reliability in the results' comparison.

A growth cycle consists in $15 \mathrm{~min}$ of immersion in a $1 \mathrm{mM}$ copper acetate hexahydrate solution, $\mathrm{Cu}(\mathrm{OAc})_{2} \cdot 6 \mathrm{H}_{2} \mathrm{O}$ followed by $3 \mathrm{~min}$ of rinsing in pure ethanol, followed by $30 \mathrm{~min}$ of immersion in a $0.2 \mathrm{mM}$ solution of trimesic acid, and ended by $3 \mathrm{~min}$ of rinsing in pure ethanol. The reagent solutions were kept at $50{ }^{\circ} \mathrm{C}$. Each sample went through 15 cycles of growth.

X-ray Photoelectron Spectroscopy. XPS spectra were obtained on a homebuilt system from the Zernike Institute for Advanced Material at the University of Groningen (The Netherlands) with a VG Microtech CLAM 2 hemispherical analyzer and a non-monochromatic $\mathrm{Al} \mathrm{K} \alpha$ source $\left(1486.6 \mathrm{eV}\right.$, operating at $10 \mathrm{kV}, 34 \mathrm{~mA}, 30^{\circ}$ source). The fittings of the spectra were done using the Unifit software version 2018 .

Profilometer. The thickness measurements of the SURMOF layers for the growth curves (SURMOF thickness as a function of the initial EUV dose) were obtained using a KLA-Tencor alpha-step 500 profilometer.

Static Water Angle Contact. The water contact angle (WCA) pictures were taken using a homebuilt system. Images were taken using an Apple camera and with the software miXscope. Image analysis was conducted with the software imageJ using the drop analysis LB-ADSA plug-in.

\section{ASSOCIATED CONTENT}

\section{SI Supporting Information}

The Supporting Information is available free of charge at https://pubs.acs.org/doi/10.1021/acsami.1c13667.

Macrophotographs of the hydrophobicity test, open frame patterns and patterns post etching; SEM images of line/space patterns, open-frame patterns and open frame patterns post etching; and a graph depicting the Fluorine content in the samples post EUV exposure and post exchange procedure as obtained from XPS analysis (PDF)

\section{Hydrophobicity test (MOV)}




\section{AUTHOR INFORMATION}

\section{Corresponding Authors}

O. Lugier - Advanced Research Center for Nanolithography, 1098XG Amsterdam, The Netherlands;

Email: o.c.m.lugier@uva.nl

S. Castellanos - Advanced Research Center for Nanolithography, 1098XG Amsterdam, The Netherlands; ○ orcid.org/0000-0002-4880-1910; Email: s.castellanos@ arcnl.nl

\section{Authors}

N. Thakur - Advanced Research Center for Nanolithography, 1098XG Amsterdam, The Netherlands; ㅇo orcid.org/00000001-5252-0938

L. Wu - Advanced Research Center for Nanolithography, 1098XG Amsterdam, The Netherlands; (1) orcid.org/00000002-3061-0059

M. Vockenhuber - Paul Scherrer Institute, 5232 Villigen, Switzerland

Y. Ekinci - Paul Scherrer Institute, 5232 Villigen, Switzerland

Complete contact information is available at:

https://pubs.acs.org/10.1021/acsami.1c13667

\section{Author Contributions}

O.L. performed most of the experimental work and wrote the manuscript. N.T. and L.W. participated in the EUV exposure experiments and exposed some of the samples. M.V. and Y.E. provided the setup for EUV interference lithography as well as technical assistance. S.C. designed and supervised the work, corrected the manuscript, and gave guidance for the figures' design and selection of contents.

\section{Notes}

The authors declare no competing financial interest.

\section{ACKNOWLEDGMENTS}

We acknowledge the Paul Scherrer Institute, Villigen, Switzerland, for provision of synchrotron radiation beamtime at the XIL-II beamline (proposals 20180993 and 20182117). The research leading to these results has received funding from the European Union's Horizon 2020 research and innovation program under grant agreement no. 730872, project CALIPSOplus. O.L. and S.C. thank Prof. R. Hoekstra, Dr. H. T. Jonkman, and S. Berg for the access and assistance using the XP spectrometer at the University of Groningen and Prof. A. M. Brouwer (University of Amsterdam), Dr. R. Bliem (ARCNL), and P. Kraus (ARCNL) for their kind advice and scientific discussion.

\section{REFERENCES}

(1) Moore, G. Cramming More Components onto Integrated Circuits, Reprinted from Electronics; IEEE, 1965; Vol. 38.

(2) IEEE International Roadmap for Devices and SystemsTM. https://irds.ieee.org, (accessed Jan 2, 2020).

(3) Narasimhan, A.; Wisehart, L.; Grzeskowiak, S.; Ocola, L. E.; Denbeaux, G.; Brainard, R. L. What We Don't Know About EUV Exposure Mechanisms. J. Photopolym. Sci. Technol. 2017, 30, 113120.

(4) Wood, O. R. EUVL: Challenges to Manufacturing Insertion. J. Photopolym. Sci. Technol. 2017, 30, 599-604.

(5) Xu, H.; Kosma, V.; Giannelis, E.; Ober, C. K.; Sakai, K. EUV Photolithography: Resist Progress and Challenges. In Extreme Ultraviolet (EUV) Lithography IX; Proceedings of SPIE, 2018; p 2.
(6) Levinson, H. J.; Brunner, T. A. Current Challenges and Opportunities for EUV Lithography. In International Conference on Extreme Ultraviolet Lithography 2018; Proceedings of SPIE, 2018; p 2.

(7) Li, L.; Liu, X.; Pal, S.; Wang, S.; Ober, C. K.; Giannelis, E. P. Extreme Ultraviolet Resist Materials for Sub-7 Nm Patterning. Chem. Soc. Rev. 2017, 46, 4855-4866.

(8) Fallica, R.; Rezvani, S. J.; Nannarone, S.; Borisov, S.; De Simone, D.; Babin, S.; Lorusso, G.; Vandenberghe, G. The Hidden Energy Tail of Low Energy Electrons in EUV Lithography. In Advances in Patterning Materials and Processes XXXVI; Proceedings of SPIE, 2019; p 8.

(9) Ashby, P. D.; Olynick, D. L.; Ogletree, D. F.; Naulleau, P. P. Resist Materials for Extreme Ultraviolet Lithography: Toward LowCost Single-Digit-Nanometer Patterning. Adv. Mater. 2015, 27, 5813-5819.

(10) Bilski, B.; Zimmermann, J.; Roesch, M.; Liddle, J.; van Setten, E.; Bottiglieri, G.; van Schoot, J. High-NA EUV Imaging: Challenges and Outlook. In 35th European Mask and Lithography Conference (EMLC 2019); Behringer, U. F., Finders, J., Eds.; SPIE, 2019; p 42.

(11) Zahlten, C.; Gräupner, P.; van Schoot, J.; Kuerz, P.; Stoeldraijer, J.; Kaiser, W. High-NA EUV Lithography: Pushing the Limits. In 35th European Mask and Lithography Conference (EMLC 2019); Behringer, U. F., Finders, J., Eds.; SPIE, 2019; p 43.

(12) Henke, B. L.; Gullikson, E. M.; Davis, J. C. X-Ray Interactions: Photoabsorption, Scattering, Transmission, and Reflection at $\mathrm{E}=50$ 30,000 EV, Z = 1-92. At. Data Nucl. Data Tables 1993, 54, 181-342.

(13) Frey, S.; Heister, K.; Zharnikov, M.; Grunze, M. Modification of Semifluorinated Alkanethiolate Monolayers by Low Energy Electron Irradiation. Phys. Chem. Chem. Phys. 2000, 2, 1979-1987.

(14) Zharnikov, M.; Frey, S.; Heister, K.; Grunze, M. Modification of Alkanethiolate Monolayers by Low Energy Electron Irradiation: Dependence on the Substrate Material and on the Length and Isotopic Composition of the Alkyl Chains. Langmuir 2000, 16, 26972705.

(15) Harnett, C. K.; Satyalakshmi, K. M.; Craighead, H. G. LowEnergy Electron-Beam Patterning of Amine-Functionalized SelfAssembled Monolayers. Appl. Phys. Lett. 2000, 76, 2466-2468.

(16) Yildirim, C.; Füser, M.; Terfort, A.; Zharnikov, M. Modification of Aromatic Self-Assembled Monolayers by Electron Irradiation: Basic Processes and Related Applications. J. Phys. Chem. C 2017, 121, 567576.

(17) Smith, R. K.; Lewis, P. A.; Weiss, P. S. Patterning SelfAssembled Monolayers. Prog. Surf. Sci. 2004, 75, 1-68.

(18) Szymonik, M.; Davies, A. G.; Wälti, C. DNA Self-AssemblyDriven Positioning of Molecular Components on Nanopatterned Surfaces. Nanotechnology 2016, 27, 395301.

(19) Shaali, M.; Woller, J. G.; Johansson, P. G.; Hannestad, J. K.; De Battice, L.; Aissaoui, N.; Brown, T.; El-Sagheer, A. H.; Kubatkin, S.; Lara-Avila, S.; Albinsson, B.; Jesorka, A. Site-Selective Immobilization of Functionalized DNA Origami on Nanopatterned Teflon AF. J. Mater. Chem. C 2017, 5, 7637-7643.

(20) Lee, S. H.; Rho, W.-Y.; Park, S. J.; Kim, J.; Kwon, O. S.; Jun, B.H. Multifunctional Self-Assembled Monolayers via Microcontact Printing and Degas-Driven Flow Guided Patterning. Sci. Rep. 2018, $8,1-8$.

(21) Yang, J.; Ichii, T.; Murase, K.; Sugimura, H. Site-Selective Assembly and Reorganization of Gold Nanoparticles along Aminosilane-Covered Nanolines Prepared on Indium-Tin Oxide. Langmuir 2012, 28, 7579-7584.

(22) Ito, H.; Iio, A.; Tokuhara, K.; Sakaue, H.; Kadoya, Y.; Suzuki, H. Estimation of the Number of Quantum Dots Immobilized on an Ultra-Flat Au Surface. Nanoscale Res. Lett. 2017, 12, 301.

(23) Wu, C.; Wang, Y.; Han, X.; Hu, X.; Cheng, Q.; Han, B.; Liu, Q.; Ren, T.; He, Y.; Sun, S.; Ma, H. Site-Selective Assembly of Quantum Dots on Patterned Self-Assembled Monolayers Fabricated by Laser Direct-Writing. Nanotechnology 2012, 23, 235302.

(24) Zhuang, J.-L.; Terfort, A.; Wöll, C. Formation of Oriented and Patterned Films of Metal-Organic Frameworks by Liquid Phase Epitaxy: A Review. Coord. Chem. Rev. 2016, 307, 391-424. 
(25) Wang, N.; Cheng, L.; Si, J.; Liang, X.; Jin, Y.; Wang, J.; Huang, W. Morphology Control of Perovskite Light-Emitting Diodes by Using Amino Acid Self-Assembled Monolayers. Appl. Phys. Lett. 2016, $108,141102$.

(26) Kim, G.; An, S.; Hyeong, S.-K.; Lee, S.-K.; Kim, M.; Shin, N. Perovskite Pattern Formation by Chemical Vapor Deposition Using Photolithographically Defined Templates. Chem. Mater. 2019, 31, $8212-8221$.

(27) Castellanos, S.; Verhoeven, J.; Frenken, J.; Antonov, P.; Ten kate, N.; Lugier, O. Method and Apparatus for Forming a Patterned Layer of Carbon, Method of Forming a Patterned Layer of Material. WO2019166409A1, 2019.

(28) Heinke, L.; Wöll, C. Surface-Mounted Metal-Organic Frameworks: Crystalline and Porous Molecular Assemblies for Fundamental Insights and Advanced Applications. Adv. Mater. 2019, 31, 1806324.

(29) Semrau, A. L.; Zhou, Z.; Mukherjee, S.; Tu, M.; Li, W.; Fischer, R. A. Surface-Mounted Metal-Organic Frameworks: Past, Present, and Future Perspectives. Langmuir 2021, 37, 6847-6863.

(30) Lugier, O.; Troglia, A.; Sadegh, N.; van Kessel, L.; Bliem, R.; Mahne, N.; Nannarone, S.; Castellanos, S. Extreme Ultraviolet Photoelectron Spectroscopy on Fluorinated Monolayers: Towards Nanolithography on Monolayers. J. Photopolym. Sci. Technol. 2020, 33, 229-234.

(31) Srisombat, L.; Jamison, A. C.; Lee, T. R. Stability: A Key Issue for Self-Assembled Monolayers on Gold as Thin-Film Coatings and Nanoparticle Protectants. Colloids Surf., A 2011, 390, 1-19.

(32) Baralia, G. G.; Duwez, A.-S.; Nysten, B.; Jonas, A. M. Kinetics of Exchange of Alkanethiol Monolayers Self-Assembled on Polycrystalline Gold. Langmuir 2005, 21, 6825-6829.

(33) Chidsey, C. E. D.; Loiacono, D. N. Chemical Functionality in Self-Assembled Monolayers: Structural and Electrochemical Properties. Langmuir 1990, 6, 682-691.

(34) Ford, K.; Battersby, B. J.; Wood, B. J.; Gentle, I. R. The Production and Verification of Pristine Semi-Fluorinated Thiol Monolayers on Gold. J. Colloid Interface Sci. 2012, 370, 162-169.

(35) Barriet, D.; Yam, C. M.; Shmakova, O. E.; Jamison, A. C.; Lee, T. R. 4-Mercaptophenylboronic Acid SAMs on Gold: Comparison with SAMs Derived from Thiophenol, 4-Mercaptophenol, and 4Mercaptobenzoic Acid. Langmuir 2007, 23, 8866-8875.

(36) Wang, Z.; Wöll, C. Fabrication of Metal-Organic Framework Thin Films Using Programmed Layer-by-Layer Assembly Techniques. Adv. Mater. Technol. 2019, 4, 1800413.

(37) Bradshaw, D.; Garai, A.; Huo, J. Metal-Organic Framework Growth at Functional Interfaces: Thin Films and Composites for Diverse Applications. Chem. Soc. Rev. 2012, 41, 2344-2381.

(38) Li, Q.; Gies, J.; Yu, X. J.; Gu, Y.; Terfort, A.; Kind, M. Concentration-Dependent Seeding as a Strategy for Fabrication of Densely Packed Surface-Mounted Metal-Organic Frameworks (SURMOF) Layers. Chem.-Eur. J. 2020, 26, 5185-5189.

(39) Zharnikov, M.; Geyer, W.; Gölzhäuser, A.; Frey, S.; Grunze, M. Modification of Alkanethiolate Monolayers on Au-Substrate by Low Energy Electron Irradiation: Alkyl Chains and the S/Au Interface. Phys. Chem. Chem. Phys. 1999, 1, 3163-3171.

(40) Biemmi, E.; Scherb, C.; Bein, T. Oriented Growth of the Metal Organic Framework $\mathrm{Cu} 3(\mathrm{BTC}) 2(\mathrm{H} 2 \mathrm{O}) 3 \cdot \mathrm{xH} 2 \mathrm{O}$ Tunable with Functionalized Self-Assembled Monolayers. J. Am. Chem. Soc. 2007, 129, 8054-8055.

(41) Tanuma, S.; Powell, C. J.; Penn, D. R. Calculations of Electron Inelastic Mean Free Paths (IMFPS). IV. Evaluation of Calculated IMFPs and of the Predictive IMFP Formula TPP-2 for Electron Energies between 50 and 2000 EV. Surf. Interface Anal. 1993, 20, 7789.

(42) Naumkin, A.; Kraut-Vass, A.; Gaarenstroom, S.; Powell, C. NIST X-ray Photoelectron Spectroscopy Database. https://srdata. nist.gov/xps/Default.aspx (accessed Jan 1, 2017).

(43) Lio, A. EUV Photoresists: A Progress Report and Future Prospects. Synchrotron Radiat. News 2019, 32, 9-14.
(44) De Simone, D.; Vanelderen, P.; Vandenberghe, G. Photo Material Readiness at the Eve of EUVL HVM. J. Photopolym. Sci. Technol. 2017, 30, 613-617.

(45) Lugier, O.; Pokharel, U.; Castellanos, S. Impact of Synthetic Conditions on the Morphology and Crystallinity of FDMOF-1 $(\mathrm{Cu})$ Thin Films. Cryst. Growth Des. 2020, 20, 5302-5309.

(46) Vericat, C.; Vela, M. E.; Benitez, G.; Carro, P.; Salvarezza, R. C. Self-Assembled Monolayers of Thiols and Dithiols on Gold: New Challenges for a Well-Known System. Chem. Soc. Rev. 2010, 39, 1805-1834.

(47) Gronheid, R.; Solak, H. H.; Ekinci, Y.; Jouve, A.; Van Roey, F. Characterization of Extreme Ultraviolet Resists with Interference Lithography. Microelectron. Eng. 2006, 83, 1103-1106. 